\title{
A New Minimal Point Theorem in Product Spaces
}

\author{
A. Göpfert, Chr. Tammer and C. Zălinescu
}

\begin{abstract}
We derive a minimal point theorem for a subset $A$ in a cone in product spaces under a weak assumption concerning the boundedness of the considered set $A$. Using this result we improve two vectorial variants of Ekeland's variational principle. Finally, a new characterization of well-based cones is given.
\end{abstract}

Keywords: Minimal point theorems, Ekeland's variational principle, well-based cones AMS subject classiffcation: $49 \mathrm{~J} \mathrm{40,90} \mathrm{C} \mathrm{29,46} \mathrm{A} \mathrm{40,} 90 \mathrm{C} 26$

Assume that $(X, d)$ is a complete metric space, $Y$ is a separated locally convex space, $Y^{*}$ is its topological dual, $K \subset Y$ is a convex cone, i.e. $K+K \subset K$ and $[0, \infty) \cdot K \subset K$,

$$
K^{+}=\left\{y^{*} \in Y^{*}:\left\langle y, y^{*}\right\rangle \geq 0 \text { for all } y \in K\right\}
$$

is the dual cone of $K$ and

$$
K^{\#}=\left\{y^{*} \in Y^{*}:\left\langle y, y^{*}\right\rangle>0 \text { for all } y \in K \backslash\{0\}\right\} .
$$

In this note we suppose that $K$ is pointed, i.e. $K \cap(-K)=\{0\}$. The cone $K$ determines an order relation on $Y$, denoted in the sequel by $\leq_{K}$; so, for $y_{1}, y_{2} \in Y, y_{1} \leq_{K} y_{2}$ if $y_{2}-y_{1} \in K$. It is well known that " $\leq_{K}$ " is reflexive, transitive and antisymmetric. Let $k^{0} \in K \backslash\{0\}$; using the element $k^{0}$ we introduce an order relation on $X \times Y$, denoted by " $\swarrow_{k}$ ", in the following manner:

$$
\left(x_{1}, y_{1}\right) \preceq_{k^{\circ}}\left(x_{2}, y_{2}\right) \quad \text { iff } \quad y_{1}+k^{0} d\left(x_{1}, x_{2}\right) \leq_{K} y_{2} .
$$

Note that " $\mathfrak{k}^{\circ}$ " is reflexive, transitive and antisymmetric. That is, our notations are those of [3].

The essential idea for the derivation of a minimal point theorem (cf. $[2,8]$ ) in general product spaces $X \times Y$, as well as of the vectorial Ekeland principle, consists in including the ordering cone $K \subset Y$ in a "larger" cone $B \subset Y: K \backslash\{0\} \subset$ int $B$. We will use $B$ to define a suitable functional $z_{B}: Y \rightarrow \mathbb{R}$. Moreover, we will replace the usual boundedness condition of the projection $P_{Y} A$ of $A$ onto $Y$ by a weaker one.

\footnotetext{
A. Göpfert: Dept. Math. \& Inform. Univ., D-06099 Halle Chr. Tammer: Dept. Math. \& Inform. Univ., D-06099 Halle

C. Zălinescu: Fac. Math. Univ. "Al. I. Cuza", 6600 Iaşi, Romania
} 
Theorem 1. Assume that there exists a proper convex cone $B \subset Y$ such that $K \backslash\{0\} \subset$ int $B$. Suppose that the set $A \subset X \times Y$ satisfies the condition

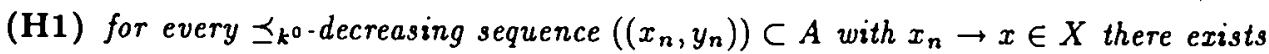
$y \in Y$ such that $(x, y) \in A$ and $(x, y) \preceq_{k^{0}}\left(x_{n}, y_{n}\right)$ for every $n \in \mathbb{N}$

and that $P_{Y}(A) \cap(\tilde{y}-$ int $B)=\emptyset$ for some $\tilde{y} \in Y$. Then for every $\left(x_{0}, y_{0}\right) \in A$ there exists $(\bar{x}, \bar{y}) \in A$, minimal with respect to $\preceq_{k^{\circ}}$, such that $(\bar{x}, \bar{y}) \preceq_{k} \circ\left(x_{0}, y_{0}\right)$.

Proof. Let

$$
z_{B}: Y \rightarrow \mathbb{R}, \quad z_{B}(y)=\inf \left\{t \in \mathbb{R}: y \in t k^{0}-\operatorname{cl} B\right\} .
$$

By [3: Lemma 7], $z_{B}$ is a continuous sublinear function such that $z_{B}\left(y+t k^{0}\right)=z_{B}(y)+t$ for all $t \in \mathbb{R}$ and $y \in Y$, and for every $\lambda \in \mathbb{R}$

$$
\begin{aligned}
& \left\{y \in Y: z_{B}(y) \leq \lambda\right\}=\lambda k^{0}-\operatorname{cl} B \\
& \left\{y \in Y: z_{B}(y)<\lambda\right\}=\lambda k^{0}-\operatorname{int} B .
\end{aligned}
$$

Moreover, if $y_{2}-y_{1} \in K \backslash\{0\}$, then $z_{B}\left(y_{1}\right)<z_{B}\left(y_{2}\right)$. Observe that for $(x, y) \in A$ we have that $z_{B}(y-\tilde{y}) \geq 0$. Otherwise for some $(x, y) \in A$ we have $z_{B}(y-\widetilde{y})<0$. It follows that there exists $\lambda>0$ such that $y-\tilde{y} \in-\lambda k^{0}-\operatorname{cl} B$. Hence

$$
y \in \tilde{y}-\left(\lambda k^{0}+\operatorname{cl} B\right) \subset \tilde{y}-(\operatorname{int} B+\operatorname{cl} B) \subset \tilde{y}-\operatorname{int} B
$$

which is a contradiction. Since $0 \leq z_{B}(y-\widetilde{y}) \leq z_{B}(y)+z_{B}(-\widetilde{y})$, it follows that $z_{B}$ is bounded from below on $P_{Y}(A)$. Let us construct a sequence $\left(\left(x_{n}, y_{n}\right)\right)_{n \geq 0} \subset A$ as follows: having $\left(x_{n}, y_{n}\right) \in A$ we take $\left(x_{n+1}, y_{n+1}\right) \in A,\left(x_{n+1}, y_{n+1}\right) \preceq_{k^{0}}\left(x_{n}, y_{n}\right)$, such that

$$
z_{B}\left(y_{n+1}\right) \leq \inf \left\{z_{B}(y):(x, y) \in A \text { and }(x, y) \preceq_{k^{\circ}}\left(x_{n}, y_{n}\right)\right\}+\frac{1}{n+1} .
$$

Of course, the sequence $\left(\left(x_{n}, y_{n}\right)\right)$ is $\preceq_{k^{0}}$-decreasing. It follows that

$$
y_{n+p}+k^{0} d\left(x_{n+p}, x_{n}\right) \leq_{K} y_{n} \quad \forall n, p \in \mathbb{N}^{*}
$$

so that

$$
d\left(x_{n+p}, x_{n}\right) \leq z_{B}\left(y_{n}\right)-z_{B}\left(y_{n+p}\right) \leq \frac{1}{n} \quad \forall n, p \in \mathbb{N}^{*} .
$$

It follows that $\left(x_{n}\right)$ is a Cauchy sequence in the complete metric space $(X, d)$, and so $\left(x_{n}\right)$ is convergent to some $\bar{x} \in X$. By condition (H1) there exists $\bar{y} \in Y$ such that $(\bar{x}, \bar{y}) \in A$ and $(\bar{x}, \bar{y}) \preceq_{k^{\circ}}\left(x_{n}, y_{n}\right)$ for every $n \in \mathbb{N}$.

Let us show that $(\bar{x}, \bar{y})$ is the desired element. Indeed, $(\vec{x}, \bar{y}) \preceq_{k^{\circ}}\left(x_{0}, y_{0}\right)$. Suppose that $\left(x^{\prime}, y^{\prime}\right) \in A$ is such that $\left(x^{\prime}, y^{\prime}\right) \preceq_{k^{\circ}}(\bar{x}, \bar{y}) \quad\left(\preceq_{k^{\circ}}\left(x_{n}, y_{n}\right)\right.$ for every $\left.n \in \mathbb{N}\right)$. Thus $z_{B}\left(y^{\prime}\right)+d\left(x^{\prime}, \bar{x}\right) \leq z_{B}(\bar{y})$, whence

$$
d\left(x^{\prime}, \bar{x}\right) \leq z_{B}(\bar{y})-z_{B}\left(y^{\prime}\right) \leq z_{B}\left(y_{n}\right)-z_{B}\left(y^{\prime}\right) \leq \frac{1}{n} \quad \forall n \geq 1 .
$$

It follows that $d\left(x^{\prime}, \bar{x}\right)=z_{B}(\bar{y})-z_{B}\left(y^{\prime}\right)=0$. Hence $x^{\prime}=\bar{x}$. As $y^{\prime} \leq_{K} \bar{y}$, if $y^{\prime} \neq \bar{y}$, then $\bar{y}-y^{\prime} \in K \backslash\{0\}$, whence $z_{B}\left(y^{\prime}\right)<z_{B}(\bar{y})$, which is a contradiction. Therefore $\left(x^{\prime}, y^{\prime}\right)=(\bar{x}, \bar{y})$ 
Comparing with [3: Theorem 4], note that the present condition on $K$ is stronger (because in this case $K^{\#} \neq \emptyset$ ), while the condition on $A$ is weaker ( $A$ may be not contained in a half-space). Note that when $K$ and $k^{0}$ are as in Theorem 1 , Corollaries 2 and 3 from [3] may be improved. In the next result $Y^{\bullet}=Y \cup\{\infty\}$ with $\infty \notin Y$; we consider that $y \leq K \infty$ for every $y \in Y$. We consider also a function $f: X \rightarrow Y^{\bullet}$ and $\operatorname{dom} f=\{x \in X: f(x) \neq \infty\}$.

In the following corollary we derive a variational principle of Ekeland's type for objective functions which take values in a general space $Y$ (cf. $[2,3,5-7]$ ) under a weaker assumption with respect to the usual lower semicontinuity. For the case $Y=\mathbb{R}$, assumption (H4) in Corollary 2 is fulfilled for decreasingly semicontinuous real-valued functions as in the paper [4].

Corollary 2. Let $f: X \rightarrow Y^{\bullet}$. Assume that there exists a proper convex cone $B \subset Y$ such that $K \backslash\{0\} \subset$ int $B$ and $f(X) \cap(\tilde{y}-B)=\emptyset$ for some $\tilde{y} \in Y$. Also, suppose that

(H3) $\left\{x^{\prime} \in X: f\left(x^{\prime}\right)+k^{0} d\left(x^{\prime}, x\right) \leq_{K} f(x)\right\}$ is closed for every $x \in X$

or

(H4) for every sequence $\left(x_{n}\right) \subset \operatorname{dom} f$ with $x_{n} \rightarrow x$ and $\left(f\left(x_{n}\right)\right) \leq_{K}$-decreasing, $f(x) \leq K f\left(x_{n}\right)$ for every $n \in \mathbb{N}$, and $K$ is closed in the direction $k^{0}$.

Then for every $x_{0} \in \operatorname{dom} f$ there exists $\bar{x} \in X$ such that

$$
f(\bar{x})+k^{0} d\left(\bar{x}, x_{0}\right) \leq_{K} f\left(x_{0}\right)
$$

and

$$
\forall x \in X: \quad f(x)+k^{0} d(\bar{x}, x) \leq_{K} f(\bar{x}) \quad \Longrightarrow \quad x=\bar{x} .
$$

We say that $K$ is closed in the direction $k^{0}$.if $K \cap\left(y-\mathbb{R}_{+} k^{0}\right)$ is closed for every $y \in K$. The proof of Corollary 2 is similar to those of Corollaries 2 and 3 in [3].

As mentioned in [3], condition (H1) is verified if $K$ is a well based convex cone, $Y$ is a Banach space and $A$ is closed. As usually (cf. [1]), a convex set $S$ is said to be a base for a convex cone $K \subset Y$ if

$$
K=\mathbb{R}_{+} S=\{\lambda y: \lambda \geq 0 \text { and } y \in S\} \quad \text { and } \quad 0 \notin \mathrm{cl} S .
$$

The cone $K$ is called well based if $K$ has a bounded base $S$. Concerning well based convex cones in normed spaces we have the following characterization.

Proposition 3. Let $Y$ be a normed vector space and $K \subset Y$ a proper convex cone. Then $K$ is well based if and only if there exist $k^{0} \in K$ and $z^{*} \in K^{+}$such that $\left\langle k^{0}, z^{*}\right\rangle>0$ and

$$
K \cap S_{1} \subset k^{0}+\left\{y \in Y:\left\langle y, z^{*}\right\rangle>0\right\}
$$

where $S_{1}=\{y \in Y:\|y\|=1\}$ is the unit sphere in $Y$.

Proof. Suppose first that $K$ is well based with bounded base $S$; therefore $0 \notin \operatorname{cl} S$ and $K=[0, \infty) \cdot S$. Then there exists $z^{*} \in Y^{*}$ such that $1 \leq\left\langle y, z^{*}\right\rangle$ for all $y \in S$. Consider $\widetilde{S}:=\left\{k \in K^{\prime}:\left\langle k, z^{*}\right\rangle=1\right\}$. It follows that $\widetilde{S}$ is a base of $K$; moreover, since 
$\tilde{S} \subset[0,1] \cdot S, \tilde{S}$ is also bounded. Taking $k^{1} \in K \backslash\{0\}$ we have $K \cap S_{1} \subset \lambda k^{1}+B_{+}$for some $\lambda>0$, where $B_{+}=\left\{y \in Y:\left\langle y, z^{*}\right\rangle>0\right\}$. Otherwise

$$
\forall n \in \mathbb{N}^{*} \exists k_{n} \in K \cap S_{1}: \quad k_{n} \notin \frac{1}{n} k^{1}+B_{+} .
$$

Therefore $\left\langle k_{n}, z^{*}\right\rangle \leq \frac{1}{n}\left\langle k^{1}, z^{*}\right\rangle$ for every $n \geq 1$. But, because $\widetilde{S}$ is a base, $k_{n}=\lambda_{n} b_{n}$ with $\lambda_{n}>0$ and $b_{n} \in \widetilde{S}_{\text {; }}$ it follows that $1=\left\|k_{n}\right\|=\lambda_{n}\left\|b_{n}\right\| \leq \lambda_{n} M$ with $M>0$ (because $\widetilde{S}$ is bounded). Therefore

$$
M^{-1} \leq \lambda_{n}=\left\langle\lambda_{n} b_{n}, z^{*}\right\rangle=\left\langle k_{n}, z^{*}\right\rangle \leq n^{-1}\left\langle k^{1}, z^{*}\right\rangle \quad \forall n \in \mathbb{N}^{*}
$$

whence $M^{-1} \leq 0$, which is a contradiction. Thus there exists $\lambda>0$ such that $K \cap S_{1} \subset$ $\lambda k^{1}+B_{+}$. Taking $k^{0}:=\lambda k^{1}$ the conclusion follows.

Suppose now that $K \cap S_{1} \subset k^{0}+B_{+}$for some $k^{0} \in K$ and $z^{*} \in K^{+}$with $\left\langle k^{0}, z^{*}\right\rangle=$ $c>0$, where $B_{+}$is defined as above. Consider $S=\left\{k \in K:\left\langle k, z^{*}\right\rangle=1\right\}$. Let $k \in K \backslash\{0\}$. Then $\|k\|^{-1} k=k^{0}+y$ for some $y \in B_{+}$. It follows that $\left\langle k, z^{*}\right\rangle>c\|k\|>0$; therefore $z^{*} \in K^{\#}$ and so $k \in(0, \infty) \cdot S$. Since $\operatorname{cl} S \subset\left\{y \in Y:\left\langle k, z^{*}\right\rangle=1\right\}$, we have that $S$ is a base of $K$. Let now $y \in S(\subset K)$. Then $\|y\|^{-1} y \in K \cap S_{1}$. There exists $z \in B_{+}$such that $\|y\|^{-1} y=k^{0}+z$. We get

$$
1=\left\langle y, z^{*}\right\rangle=\|y\|\left\langle k^{0}+z, z^{*}\right\rangle \geq c\|y\|
$$

whence $\|y\| \leq c^{-1}$. Therefore $S$ is bounded, and so $K$ is well-based

\section{References}

[1] Borwein, J. M.: Continuity and differentiability properties of convex operators. Proc. London Math. Soc. 44 (1982), 420 - 444.

[2] Göpfert, A. and Chr. Tammer: A new maximal point theorem. Z. Anal. Anw. 14 (1995), $379-390$.

[3] Göpfert, A., Tammer, Chr. and C. Zălinescu: On the vectorial Ekeland's variational principle and minimal points in product spaces. Nonlin. Anal.: Theory, Methods and Appl. (to appear).

[4] Gajek, L. and D. Zagrodny: Geometric Variational Principle. Diss. Math. 340 (1995), $55-71$.

[5] Isac, G.: The Ekeland's principle and the Pareto $\varepsilon$-efficiency. In: Multi-objective Programming and Goal Programming. Theory and Applications (ed.: M. Tamiz; Lect. Notes Econ. Math. Syst.: Vol. 432). Berlin: Springer-Verlag 1996, pp. $148-163$.

[6] Khanh, P. O.: On Caristi-Kirk's theorem and Ekeland's variational principle for Pareto extrema. Preprint. Warsaw: Polish Acad. Sci., Inst. Math., Preprint 357 (1986), 1 - 7.

[7] Nemeth, A. B.: A nonconvex vector minimization problem. Nonlin. Anal.: Theory, Methods and Appl. 10 (1986), 669 - 678.

[8] Phelps, R. R.: Convex Functions, Monotone Operators and Differentiability, 2nd Ed. Lect. Notes Math. 1364 (1993). 